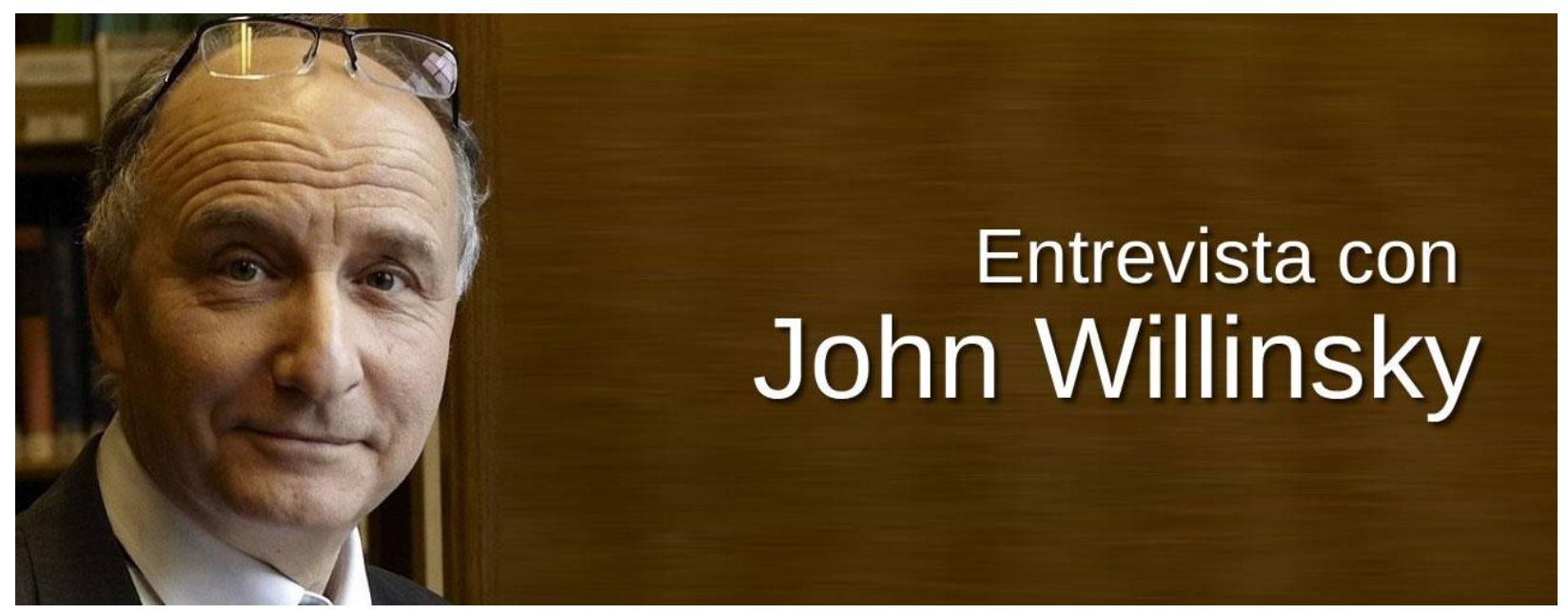

\title{
Desarrollando la Editoración Científica en América Latina
}

\section{INTERVIEW}

\section{Resumen}

Entrevista a John Willinsky, docente e investigador de la Escuela de Educación de la Standford Universty y fundador representante del Public Knowledge Project - PKP.

Palabras-clave

John Willinsky ; Public Knowledge Project ; PKP ; Editoración científica ; Comunicación científica ; Revistas científicas ; Acceso abierto ; América Latina ; Entrevistas

\section{Developing the Scholarly Publishing in Latin America}

\section{Abstract}

Interview with John Willinsky, professor and researcher at the School of Education from the Stanford University and founder of the Public Knowledge Project - PKP.

\section{Keywords}

John Willinsky ; Public Knowledge Project ; PKP ; Scholalrly publishing ; Scholarly communication; Scientific journals ; Open access ; Latin America ; Interviews

En los últimos años en América Latina se vienen desarrollando diversas iniciativas en el ámbito de la comunicación científica con el propósito de mejorar la difusión de la producción científica y sobre todo su visibilidad. Diversos proyectos se vienen articulando con este propósito, como es el caso del Public Knowledge Project - PKP, quien a través del desarrollo de herramientas de software libre viene apoyando los procesos de editoración científica de un gran número de revistas científicas en la región.

La herramienta Open Journal Systems por ejemplo cuenta con no menos de 3600 usuarios lo cual representan un número no menor de revistas científicas gestionadas por esta aplicación de reconocido uso para el proceso de gestión editorial. 
BIBLIOS converso com John Willinsky docente e investigador de la Escuela de Educación de la Standford Universty y fundador representante del PKP, quien desde hace algunos años viene desarrollando un conjunto de investigaciones sobre el desarrollo y evolución de la revistas científicas en nuestras región, así como el impacto y uso de herramientas de acceso abierto como el Open Journal Systems.

\section{Usted há declarado recientemente que América Latina es el continente del acceso abierto a la información científica, en que basa esta afirmación?}

Willinsky: Es un fenómeno muy interesante que América Latina haya optado, casi en su totalidad, por la editoración científica de libre acceso, pasando rápidamente a la editoración en línea, e incrementando al mismo tiempo el acceso abierto.

Si buscamos una explicación a ello, pienso que hay transparencia y seriedad en la editoración en América Latina. Tienen el deseo de compartir sus conocimientos con el mundo y existe una preocupación por no quedar excluidos y ser parte del circuito mundial de comunicación científica.

Al valorar el acceso abierto en todas partes, la exclusión que privilegia la investigación del norte, puede ser superada por la editoración en acceso abierto y la editoración en línea, y así todos tendrían acceso a la investigación de América Latina y esta estaría siempre disponible para todo el mundo.

Creo que es más que nada un compromiso por compartir el conocimiento. Esto explica por qué América Latina fue la primera región del mundo en utilizar en gran número un sistema de editoración abierta. América Latina ha adoptado ampliamente la editoración en acceso abierto así como el software de código abierto.

\section{Cual há sido el rol de Brasil en todo este proceso?}

Willinsky: Brasil ha jugado un papel muy importante en el desarrollo del sistema Open Journal Systems - OJS desarrollado por el PKP. El OJS desde el principio se uso en Brasil y ha funcionado gracias al apoyo del Instituto Brasileiro de Informação em Ciência e Tecnologia - IBICT y al apoyo de los editores de revistas en todo el país.

Los brasileños han contribuido en gran medida al desarrollo del OJS. Comenzaron con la traducción al portugués, siendo uno de los primeros idiomas en el que OJS fue traducido, y el que se viene manteniendo en cada nueva versión de esa plataforma, además de otros sistemas como Open Conference Systems - OCS y Open Monograph Press - OMP.

Brasil también ha contribuido en el desarrollo de ese software, con nuevas ideas de implementación, sugerencias técnicas, desarrollando filtros, en términos generales: con diversas ideas para mejorar el soporte a las publicaciones provenientes de la comunidad brasileña. Brasil ha ejercido un rol importante mediante el IBICT, facilitando apoyo, como soporte de proyecto de software libre, a toda la comunidad en diversos lugares, como en África, pudiendo proveer un apoyo muy fuerte.

\section{El Open Journal Systems es una de las herramientas más conocidas del PKP en la región. Que novedades tendremos de ella?}

Willinsky: El OJS ha sido sometido a una serie de cambios y me gustaría referirme a algunos de ellos. Una de esas novedades es que el OJS es cada vez más modular, el envió, la revisión, la edición, las etapas de producción estarán disponibles como entidades separadas y las personas serán capaces de personalizarlas. Pero además la modularidad podrá funcionar adecuadamente con los sistemas de gestión de contenidos como Drupal. Tenemos un grupo de revistas que solicitan la vista del contenido en Drupal, así como la integración de algunos módulos con Drupal, sea la vista de foro, la portada pública de la revista, de tal forma que el flujo de trabajo se mantenga gestionado por el OJS en el trasfondo. 
Promoviendo el desarrollo de OJS estamos buscando el desarrollo de estadísticas a nivel de artículo mediante el incremento en el uso de XML y de algunas formas de incubación donde el autor se relacione al co-autor, además de otras formas de post publicación como: feeds, blogs, sistemas como Facebook o Mendeley, y las citas tradicionales.

En general, buscamos un OJS más flexible, que trabaje e interactué bien con otros sistemas de gestión de contenidos. Estamos buscando que la flexibilidad gire en torno a la modularidad, estamos buscando la integración, se viene un largo periodo, digitalizando a sistemas como XML, permitiendo entradas bibliográficas, verificando referencias, verificación ubicaciones, que el sistema permita a los editores trabajar con los autores, mientras los técnicos administrativos de la publicación se ocupan de los sistemas automatizados en mayor medida que antes.

\section{Qué otras novedades nos tiene previsto el PKP en breve?}

Willinsky: Existe un conjunto de proyectos en desarrollo, hablando del PKP en términos amplios. Quizás uno de los más interesantes es liberar Open Monograph Press - OMP. Este es un sistema modular diseñado para asistir la editoración de monografías y volúmenes editados. Esto permitiría a los académicos acompañar y apoyar la editoración de obras y libros amplios que estarían disponibles en impresión bajo demanda, sea como e-books, o Kindle si se publica en la plataforma de Amazon.

Como parte de esto que está relacionado con el desarrollo futuro en XML, como una forma de etiquetado y marcado de los artículos para las capas de diseños, tenemos las etapas iniciales del desarrollo de Lemon 8-XML, donde podemos marcar partes de las marcas entradas bibliográficas y etiquetas XML para que se puedan utilizar en el control de las bases de datos, las licencias patrón, y también para localizar las citas. Entonces con Open Monograph Press y XML estamos construyendo Lemon 8-XML.

Parte de esto también es el desarrollo de la Librería de trabajo PKP que incluirá los módulos basados en el diseño del OMP, y que estarán disponible en todos nuestros sistemas de tal forma que puedan obtenerse juntos los procesos de envío, revisión, edición, y las etapas de producción; lo cual permitirá personalizarlos, así como conectarlos a otros sistemas como Drupal.

\section{Qué mensaje daría a los editores científicos de América Latina?}

Willinsky: Mi mensaje personal a todos los editores de América Latina que usa OJS es GRACIAS. Estoy profundamente agradecido por su apoyo y confianza en el sistema. Este ha sido un proyecto universitario y todo aquel que se inicie en OJS nos está ayudando.

Sigan utilizando OJS, ayuden con traducciones, envíen informes de errores, hagan sugerencias, contribuyan al foro. El foro de soporte en español muy activo. Mi mensaje es en todo sentido de agradecimiento y esperanza en que continúe su apoyo y participación. OJS es un sistema distribuido y en su acceso están asociados todos los editores y los desarrolladores tanto en Latinoamérica como en todo el mundo. En ese sentido estamos juntos en esto para construir una visión alternativa de la circulación del conocimiento, para quien trabaja en la Universidad, para quienes trabajamos en la investigación académica, como algo que es cada vez más público y cada vez más importante para la calidad de la vida democrática.

\section{Bibliografia}

OJS Latin América. Regional News and Information about Open Journal Systems.

Disponible en: < http://pkp.sfu.ca/ >. Acceso en: 30 dic. 2012

PKP. Lemon8-XML.

Disponible en: < http://pkp.sfu.ca/lemon8 >. Acceso en: 30 dic. 2012

PKP. OJS: Open Journal Systems.

Disponible en: < http://pkp.sfu.ca/?q=0js >. Acceso en: 30 dic. 2012 
PKP. OJS: Open Monograph Press.

Disponible en: < http://pkp.sfu.ca/omp > Acceso en: 30 dic. 2012

PKP. Public Knowledge Project.

Disponible en: < http://pkp.sfu.ca/ > Acceso en: 30 dic. 2012

\section{Producción}

Transcripción : : Martha E. Mantilla

Traducción y edición : Julio Santillán-Aldana

\section{Agradecimientos}

Public Knowledge Project (PKP)

Instituto Brasileiro de Informação em Ciência e Tecnologia (IBICT)

Universidade de Brasília. Faculdade de. Ciência da Informação

\section{(cc) EY-NC-ND}

This work is licensed under a Creative Commons

Attribution-Noncommercial-No Derivative Works 3.0 United States License.

\section{ULIS D-Souff}

This journal is published by the University Library System of the University of Pittsburgh as part of its D-Scribe Digital Publishing Program and is cosponsored by the University of Pittsburgh Press. 